\title{
Behavioural changes during the COVID-19 pandemic: Results of a nationwide survey in Singapore
}

Victoria JE Long, ${ }^{1}$ MPH, Jean CJ Liu,,${ }^{1,2} P h D$

\begin{abstract}
Introduction: As part of infection control measures for COVID-19, individuals have been encouraged to adopt both preventive (such as handwashing) and avoidant behavioural changes (e.g. avoiding crowds). In this study, we examined whether demographics predicted the likelihood that a person would adopt these behaviours in Singapore.

Methods: A total of 1,145 participants responded to an online survey conducted between 7 March and 21 April 2020. We collected demographic information and asked participants to report which of 17 behaviour changes they had undertaken because of the COVID-19 outbreak. Regression analyses were performed to predict the number of behavioural changes (preventive, avoidant, and total) as a function of demographics. Finally, we sought to identify predictors of persons who declared that they had not undertaken any of these measures following the outbreak.

Results: Most participants (97\%) reported at least one behavioural change on account of the pandemic, with changes increasing with the number of local COVID-19 cases $(P<0.001)$. Additionally, women and those who were younger adopted more preventive behaviours (gender: $P<0.001$; age: $P=0.001$ ). Women were more likely to increase handwashing frequency, and younger individuals were more likely to wear face masks prior to legislation. Finally, women and those who were married adopted more avoidant behaviours (gender: $P<0.001$; marital status: $P<0.001$ ), with both groups avoiding crowded areas and staying home more than usual. Women also voluntarily reduced physical contact, whereas those who were married preferentially chose outdoor venues and relied on online shopping.

Conclusion: Our characterisation of behavioural changes provides a baseline for public health advisories. Moving forward, health authorities can focus their efforts on encouraging segments of the population who do not readily adopt infection control measures against COVID-19.
\end{abstract}

Ann Acad Med Singap 2021;50:222-31

Keywords: Behaviour, COVID-19, epidemic, infectious diseases, public health

\section{INTRODUCTION}

In response to the global outbreak of coronavirus disease 2019 (COVID-19), Singapore undertook a multipronged approach to contain the pandemic. Of note, when community transmission began early in the outbreak, the government started emphasising the role that individuals had to play by adopting health-preventive behaviours. ${ }^{1,2}$

In an infectious disease outbreak such as the COVID-19 pandemic, individual-level health-protective behaviours can be classified into preventive behavioursmeasures that can prevent transmission (e.g. handwashing and wearing a mask), and avoidant behavioursmeasures that decrease contact with other individuals (e.g. avoiding crowded areas). ${ }^{3}$ As COVID-19 is believed to be transmitted primarily through contact or droplet transmission, these measures can be effective in reducing the spread of the virus, particularly when pharmacological interventions are limited. ${ }^{4,5}$

For risk communication, it is useful to understand what characteristics predict whether an individual adopts health-protective behaviours. This allows public health messaging to be targeted, improving compliance in groups that may not do so as readily. For example, in the previous outbreak of severe acute respiratory syndrome (SARS), preventive and avoidant behaviours were more likely to be adopted by: women, older individuals,

\footnotetext{
${ }^{1}$ Duke-NUS Graduate Medical School, Singapore

${ }^{2}$ Division of Social Sciences, Yale-NUS College, Singapore

Correspondence: Dr Jean CJ Liu, Yale-NUS College, 28 College Ave West, \#01-501, Singapore 138533.

Email: jeanliu@yale-nus.edu.sg
} 


\section{CLINICAL IMPACT}

\section{What is New}

- In the early stage of the pandemic, most participants voluntarily adopted health-protective behaviours.

- Women and those who were married adopted the most number of behavioural changes.

- Preventive behaviours were more likely to be adopted than avoidant behaviours.

\section{Clinical Implications}

- Public health messages can be customised based on demographics (e.g. gender, marital status).

- Stronger measures (e.g. legislation) may need to be explored to increase avoidant behaviours.

and those with higher education levels. ${ }^{3}$ In the current COVID-19 outbreak, health-protective behaviours have been observed among individuals who perceive a higher risk of infection, higher disease severity, or who are afraid of getting infected. ${ }^{6-8}$ However, demographic predictors have differed between populations studied: while age and gender were linked to behavioural changes in South Korea, these associations were not found in the UK. ${ }^{7,8}$ Furthermore, no demographic predictors were identified in a study in the US, while gender-but not age - predicted behavioural changes in a cross-country survey. ${ }^{9,10}$ This heterogeneity suggests that the uptake of health-protective behaviours may be context-specific during the COVID-19 pandemic, owing perhaps to heterogeneity in the risks of infection or severe illness between countries.

To address the context-specificity of previous findings, we conducted a large-scale survey to examine how demographics predict the uptake of health-protective behaviours in Singapore. Our study was conducted from March to April 2020, a period when the country saw a rapid increase in COVID-19 cases (from 138 cases at the start of the study, to 9,125 cases at the end of the survey period).

\section{METHODS}

\section{Study design and population}

From 7 March to 21 April 2020, 1,145 participants responded to an online survey on COVID-19. ${ }^{13}$ As the inclusion criteria, participants were aged $\geq 21$ years old, and had lived in Singapore for $\geq 2$ years. Given public health concerns, participants were recruited online via advertisements placed in community chat groups (e.g.
Facebook and WhatsApp groups for residential estates, universities, or interest groups) or via paid Facebook advertisements targeting Singapore-based users. The study was approved by the Yale-NUS College Ethics Review Committee, and participants gave written consent in accordance with the Declaration of Helsinki. The questions reported in this study were part of a larger 20-minute survey exploring: behavioural and psychological responses to COVID-19; sources from which participants received COVID-19 news; and psychological well-being (https://osf.io/pv3bj). ${ }^{11}$

\section{Predictor variables}

As predictors, participants reported the following demographic details: gender, ethnicity, religion, country of birth, marital status, education, house type and household size. As behavioural changes may be influenced by the local COVID-19 situation, we also recorded the total number of local cases reported to date, and whether the country was locked down (referred to as "circuit breaker" in Singapore) when the survey was done.

\section{Outcome variables}

As the key outcome variables, participants indicated which of 17 health-protective behaviours they had voluntarily undertaken because of the pandemic (by indicating "yes" or "no" for each behaviour). Based on prior research, ${ }^{3}$ we investigated 3 preventive behaviours, asking participants whether they had: (1) washed their hands more frequently, (2) used hand sanitisers and/or (3) wore a mask in public (prior to legislation). Additionally, we investigated 14 possible avoidant behaviours, whether participants had: (1) avoided crowded areas, (2) reduced physical contact (e.g. avoided shaking hands), (3) stayed home more than usual, (4) distanced from people with flu symptoms, (5) voluntarily changed their travel plans, (6) missed or postponed social events, (7) avoided visiting hospitals and/or healthcare settings, (8) chose outdoor over indoor venues, (9) distanced from people with recent travel to outbreak countries, (10) distanced from people with possible contact with COVID-19 cases, (11) avoided places where COVID-19 cases were reported, (12) stored up more household and/or food supplies than usual, (13) relied more on online shopping (prior to shop closures), and/or (14) avoided public transport. Across the 17 items, we assigned a score of "1" for "yes" responses, and these were summed to create three scores: the total number of behavioural changes adopted (out of 17), the total number of preventive behaviours adopted (subscale score out 
of 3), and the total number of avoidant behaviours adopted (subscale score out of 14).

Finally, we included as a separate item the following statement: "I did not take any additional measures" (yes/no response). This question allowed us to identify participants who had not made any behavioural changes as a function of COVID-19: a group that may be of higher risk for transmission.

\section{Statistical analyses}

To describe participants' demographic characteristics, survey responses were summarised with counts and medians. As the primary analysis, we then ran a linear regression model with the total number of behavioural changes as the outcome measure, and participant demographics as predictors (age, gender, ethnicity, religion, country of birth, marital status, education, house type, household size, the total number of local cases reported to date, and whether the country was locked down at the time of survey completion) (Model 1). Given that prior research distinguished preventive and avoidant behaviours, ${ }^{3,12}$ we repeated the linear regression model with the total number of preventive behaviours (Model 2), and the total number of avoidant behaviours as outcomes (Model 3). Finally, using the same demographic predictors, we ran a logistic regression model to identify individuals who had made no behavioural changes (Model 4).

For linearity, the number of local COVID-19 cases was log-transformed prior to regression analyses. For each regression model, the type 1 family-wise error rate was controlled at 0.05 through Bonferroni correction (Bonferroni-adjusted alpha level of 0.05 / 23 predictors $=0.002)$. All statistical analyses were conducted using $\mathrm{R}$ version 4.0 and STATA version 12.0 (StataCorp LLC, College Station, US).

\section{RESULTS}

\section{Response rate}

Of 1,390 individuals who clicked the survey link, 1,145 $(82.4 \%)$ provided informed consent and participated in the survey. A further $192(16.77 \%)$ participants were excluded from statistical analyses as they did not complete the primary outcome measures (on behavioural changes).

As shown in Table 1, the final sample of 953 participants was comparable to the resident Singapore population in: the proportion of Singapore citizens, marital status, and household size ( $\leq 10 \%$ difference). However, the pool of respondents had a greater representation of women ( $65.1 \%$ versus $51.1 \%)$, university graduates $(72.7 \%$ vs $32.4 \%)$, persons of no religion $(28.0 \%$ vs $18.5 \%)$ or of Christian belief $(36.2 \%$ vs $18.8 \%)$. Conversely, there was a reduced representation of participants who lived in 1-3-room public housing flats (6.7\% vs 23.7\%). Survey respondents were also more likely to be of Chinese ethnicity than persons in the general population $(87.0 \%$ vs $74.3 \%)$.

\section{Overview of COVID-19 behaviour changes}

On the whole, participants adopted a median of 8 (interquartile range [IQR] 5-11) behavioural changes owing to the COVID-19 pandemic. This corresponded to a median of 2 (IQR 2-3) preventive measures, and 6 (IQR 3-8) avoidant measures.

When we accounted for the number of possible changes within each category (dividing the scores by 3 and 14, respectively), we found that participants were more likely to adopt preventive than avoidant measures (paired t-test of averaged preventive vs avoidant scores: $\mathrm{t}(952)=30.3, P<0.001)$. Only 29 participants $(3.04 \%)$ reported that they had not changed their behaviours at all.

\section{Predicting behavioural change: regression models}

In our first regression model, we sought to predict the total number of behavioural changes based on participant demographics (Table 2). We first observed that behavioural changes tracked the local COVID-19 situation: namely, as the number of local cases increased, individuals adapted their behaviours in response $(\mathrm{b}=3.03, \mathrm{t}(913)=3.96, P<0.001)$. Having controlled for local transmission, gender emerged as a significant predictor, with women adopting an average of 0.14 more changes than men $(\mathrm{t}(913)=-4.49, P<0.001)$. Being married was also associated with a higher number of health-protective behaviours than being single $(b=1.09$, $\mathrm{t}(913)=3.52, P<0.001)$.

In our second and third models, we examined whether demographic predictors differed for preventive vs avoidant behaviours. In terms of demographics, while the adoption of preventive behaviours was predicted by gender $(b=-0.241, \mathrm{t}(913)=-4.33, P<0.001)$ and age $(b=-0.008, \mathrm{t}(913)=-3.11, P=0.001)$, the adoption of avoidant behaviours was predicted by gender $(b=-0.902, \mathrm{t}(913)=-3.90, P<0.001)$ and marital status (being married vs being single; $b=0.973$, $\mathrm{t}(913)=3.45, P<0.001)$.

Finally, in our fourth model, we found that no demographic predictor significantly identified the small 
Table 1. Baseline demographics of participants

\begin{tabular}{|c|c|c|}
\hline Characteristic & $\mathbf{n}$ & $(\%)$ \\
\hline \multicolumn{3}{|l|}{ Age group $($ Mean=39.5, $\mathrm{SD}=12.6)$} \\
\hline \multicolumn{3}{|l|}{ Gender } \\
\hline Female & 620 & $(65.1)$ \\
\hline Male & 333 & $(34.9)$ \\
\hline Did not answer & 0 & \\
\hline \multicolumn{3}{|l|}{ Ethnicity } \\
\hline Chinese & 829 & $(87.0)$ \\
\hline Indian & 46 & $(4.8)$ \\
\hline Malay & 25 & $(2.6)$ \\
\hline Filipino & 15 & $(1.5)$ \\
\hline Caucasian & 13 & $(1.3)$ \\
\hline Others & 1 & $(0.1)$ \\
\hline Did not answer & 24 & $(2.5)$ \\
\hline \multicolumn{3}{|l|}{ Religion } \\
\hline Christianity (Protestant) & 345 & $(36.2)$ \\
\hline No religion & 267 & $(28.0)$ \\
\hline Buddhism & 138 & $(14.5)$ \\
\hline Roman Catholicism & 98 & $(10.2)$ \\
\hline Taoism/ Chinese traditional beliefs & 38 & $(4.0)$ \\
\hline Islam & 33 & $(3.4)$ \\
\hline Hinduism & 27 & $(2.8)$ \\
\hline Others & 6 & $(0.6)$ \\
\hline Did not answer & 1 & $(0.1)$ \\
\hline \multicolumn{3}{|l|}{ Marital status } \\
\hline Married & 534 & $(56.0)$ \\
\hline Single & 391 & $(41.0)$ \\
\hline Widowed/ separated/ divorced & 27 & $(2.8)$ \\
\hline Did not answer & 1 & $(0.1)$ \\
\hline \multicolumn{3}{|l|}{ Education level } \\
\hline 1: Primary school & 3 & $(0.3)$ \\
\hline 2: Secondary school & 44 & $(4.6)$ \\
\hline 3: Junior college & 82 & $(8.6)$ \\
\hline 4: Vocational training & 14 & $(1.4)$ \\
\hline 5: Polytechnic/ diploma & 114 & $(11.9)$ \\
\hline 6: University (undergraduate) & 486 & $(51.0)$ \\
\hline 7: University (postgraduate) & 207 & (21.7) \\
\hline Did not answer & 3 & $(0.3)$ \\
\hline
\end{tabular}

Table 1. Baseline demographics of participants (Cont'd)

\begin{tabular}{|lll}
\hline Characteristic & $\mathbf{n}$ & $\mathbf{( \% )}$ \\
\hline House type & & \\
\hline 1: HDB flat: $1-2$ rooms & 8 & $(0.8)$ \\
\hline 2: HDB flat: 3 rooms & 64 & $(6.7)$ \\
\hline 3: HDB flat: 4 rooms & 233 & $(24.4)$ \\
\hline 4: HDB flat: 5 rooms or executive flats & 265 & $(27.8)$ \\
\hline 5: Condominium or private apartments & 260 & $(27.2)$ \\
\hline 6: Landed property & 111 & $(11.6)$ \\
\hline Did not answer & 12 & $(1.2)$ \\
\hline Household size & & \\
\hline 1 & 44 & $(4.6)$ \\
\hline 2 & 124 & $(13.0)$ \\
\hline 3 & 210 & $(22.0)$ \\
\hline 4 & 292 & $(30.6)$ \\
\hline 5+ & 282 & $(29.5)$ \\
\hline Did not answer & 1 & $(0.1)$ \\
\hline Country of birth & 196 & $(20.5)$ \\
\hline Singapore & 757 & $(79.4)$ \\
\hline
\end{tabular}

proportion of individuals who had not undertaken any measures on account of COVID-19 (all $P>$ Bonferroniadjusted alpha of 0.002).

\section{Follow-up exploratory analyses}

\section{Examining whether behavioural changes tracked subjective responses to the COVID-19 situation}

To understand the regression models, we conducted exploratory analyses on variables that had emerged as significant predictors. As behavioural changes tracked the number of COVID-19 cases, we examined whether this pattern of results arose from fear of the situation. ${ }^{11}$ We found that participants' fear increased with the number of local cases (Spearman's rho $=0.18, P<0.001$ ), and that all 3 metrics of behavioural changes increased with fear levels (Spearman's rho for total: 0.36, $P<0.001$; preventive: $0.23, P<0.001$; avoidant: $0.35, P<0.001$ ).

\section{Understanding which behavioural changes differed as a function of gender, marital status and age}

We further conducted follow-up chi-square analyses on the three demographic variables that had emerged as significant predictors in the regression models. 
Table 2. Predicting behavioural changes during the COVID-19 outbreak

\begin{tabular}{|c|c|c|c|c|}
\hline & \multicolumn{4}{|c|}{ Outcome $^{\mathrm{a}}$} \\
\hline & $\begin{array}{c}\text { (1) } \\
\text { Total number of } \\
\text { behaviours }\end{array}$ & $\begin{array}{c}\text { (2) } \\
\text { Preventive } \\
\text { behaviours }\end{array}$ & $\begin{array}{c}\text { (3) } \\
\text { Avoidant } \\
\text { behaviours }\end{array}$ & $\begin{array}{c}(4) \\
\text { No behaviour } \\
\text { changes adopted }\end{array}$ \\
\hline Age & $-0.002(0.011)$ & $-0.008(0.002)^{b}$ & $0.005(0.010)$ & $0.041(0.018)$ \\
\hline \multicolumn{5}{|l|}{$\begin{array}{l}\text { Gender } \\
\text { (base }=\text { female) }\end{array}$} \\
\hline Male & $-1.14(0.254)^{b}$ & $-0.241(0.055)^{b}$ & $-0.902(0.231)^{\mathrm{b}}$ & $0.757(0.411)$ \\
\hline \multicolumn{5}{|l|}{$\begin{array}{l}\text { Ethnicity } \\
\text { (base = Chinese) }\end{array}$} \\
\hline Indian & $-1.20(1.39)$ & $-0.743(0.304)$ & $-0.463(1.26)$ & $-31.4(268)$ \\
\hline Malay & $-1.01(0.949)$ & $-0.397(0.208)$ & $-0.620(0.863)$ & $-13.4(172)$ \\
\hline Filipino & $1.04(1.10)$ & $0.430(0.242)$ & $0.617(1.00)$ & $-29.4(327)$ \\
\hline Caucasian & $-0.004(1.05)$ & $-0.318(0.796)$ & $0.313(0.962)$ & $-15.6(292)$ \\
\hline Others & $1.42(3.63)$ & $-2.21(0.085)$ & $3.64(3.30)$ & $-14.6(1080)$ \\
\hline \multicolumn{5}{|l|}{$\begin{array}{l}\text { Religion } \\
\text { (base }=\text { no religion) }\end{array}$} \\
\hline Christianity (Protestant) & $0.795(0.390)$ & $0.172(0.085)$ & $0.623(0.354)$ & $-0.542(0.518)$ \\
\hline Buddhism & $0.291(0.632)$ & $0.037(0.138)$ & $0.254(0.574)$ & $-1.20(0.816)$ \\
\hline Roman Catholicism & $0.800(1.34)$ & $0.360(0.294)$ & $0.440(1.21)$ & $-13.7(1.10)$ \\
\hline Taoism/ Chinese traditional beliefs & $1.63(1.18)$ & $-0.034(0.259)$ & $1.32(1.07)$ & $-0.555(1.10)$ \\
\hline Islam & $0.647(0.463)$ & $-0.034(0.101)$ & $0.681(0.421)$ & $15.5(172)$ \\
\hline Hinduism & $0.209(0.310)$ & $-0.014(0.400)$ & $0.223(0.282)$ & $31.3(268)$ \\
\hline Others & $-4.07(1.82)$ & $-0.629(0.400)$ & $-3.44(1.65)$ & $2.08(1.26)$ \\
\hline \multicolumn{5}{|l|}{$\begin{array}{l}\text { Marital status } \\
\text { (base }=\text { single) }\end{array}$} \\
\hline Married & $1.09(0.309)^{\mathrm{b}}$ & $0.117(0.067)$ & $0.973(0.281)^{\mathrm{b}}$ & $-0.739(0.552)$ \\
\hline Widowed/ separated/ divorced & $-0.495(0.433)$ & $0.009(0.094)$ & $-0.504(0.393)$ & $-0.750(0.850)$ \\
\hline Education level & $0.003(0.093)$ & $-0.005(0.020)$ & $0.009(0.084)$ & $-0.342(0.126)$ \\
\hline House type & $0.141(0.113)$ & $0.014(0.024)$ & $0.126(0.102)$ & $-0.064(0.185)$ \\
\hline Household size & $-0.013(0.114)$ & $0.047(0.025)$ & $-0.060(0.103)$ & $0.124(0.208)$ \\
\hline \multicolumn{5}{|l|}{$\begin{array}{l}\text { Country of birth } \\
\text { (base =Singapore) }\end{array}$} \\
\hline Other & $-0.730(0.338)$ & $-0.152(0.074)$ & $-0.578(0.307)$ & $-0.169(0.589)$ \\
\hline \multicolumn{5}{|l|}{$\begin{array}{l}\text { Lockdown } \\
(\text { base }=\text { no) }\end{array}$} \\
\hline Lockdown & $-1.76(0.017)$ & $-0.621(0.161)^{b}$ & $-1.138(0.670)$ & $-0.043(1.21)$ \\
\hline $\begin{array}{l}\text { Number of local COVID-19 cases } \\
\text { (log transformed) }\end{array}$ & $3.03(0.612)^{b}$ & $0.531(0.134)^{\mathrm{b}}$ & $2.50(0.556)^{\mathrm{b}}$ & $-0.151(0.986)$ \\
\hline$R^{2}$ & 0.123 & 0.091 & 0.124 & 0.144 \\
\hline
\end{tabular}

${ }^{a}$ Data reported as beta estimates (standard error)

${ }^{\mathrm{b}}$ Indicates significance at $P<0.002$ (following Bonferroni corrections) 
Gender. As shown in Fig. 1, women were more likely than men to: (1) wash their hands more frequently $\left(\chi^{2}(1, \mathrm{~N}=953)=22.17, P<0.001\right) ;(2)$ avoid crowded areas, $\left(\chi^{2}(1, \mathrm{~N}=953)=11.83, P=0.001\right)$; (3) reduce physical contact $\left(\chi^{2}(1, \mathrm{~N}=953)=9.28, P=0.002\right)$; and (4) stay home more than usual $\left(\chi^{2}(1, \mathrm{~N}=953)=9.79, P=0.002\right)$.

Marital status. As shown in Fig. 2, marital status was significantly associated with: avoiding crowded areas $\left(\chi^{2}(2, \mathrm{~N}=952)=26.29, P<0.001\right) ;(2)$ staying home more than usual $\left(\chi^{2}(2, \mathrm{~N}=952)=28.09, P<0.001\right)$; (3) choosing outdoor over indoor areas $\left(\chi^{2}(2, N=952)\right.$ $=33.04, P<0.001)$; and (4) relying more on online shopping $\left(\chi^{2}(2, \mathrm{~N}=952)=26.37, P<0.001\right)$. In each case, single participants were least likely to adopt these behaviours than those who were not single (married, widowed, separated or divorced).
Age. Finally, wearing a mask in public differed between age groups $\left(\chi^{2}(4, \mathrm{~N}=953)=33.32, P<0.001\right)$, with participants aged 21-30 most likely to adopt this behaviour (Fig. 3).

\section{DISCUSSION}

In this study, we documented for the first time how residents in Singapore had adapted their behaviours to minimise COVID-19 transmission. Focusing on the first phase of the pandemic, we found that the large majority of participants $(97 \%)$ had undertaken at least one infection control measure, with participants reporting an average of 8 lifestyle changes owing to the pandemic.

In terms of demographic predictors, health-preventive measures were most likely to be adopted by women

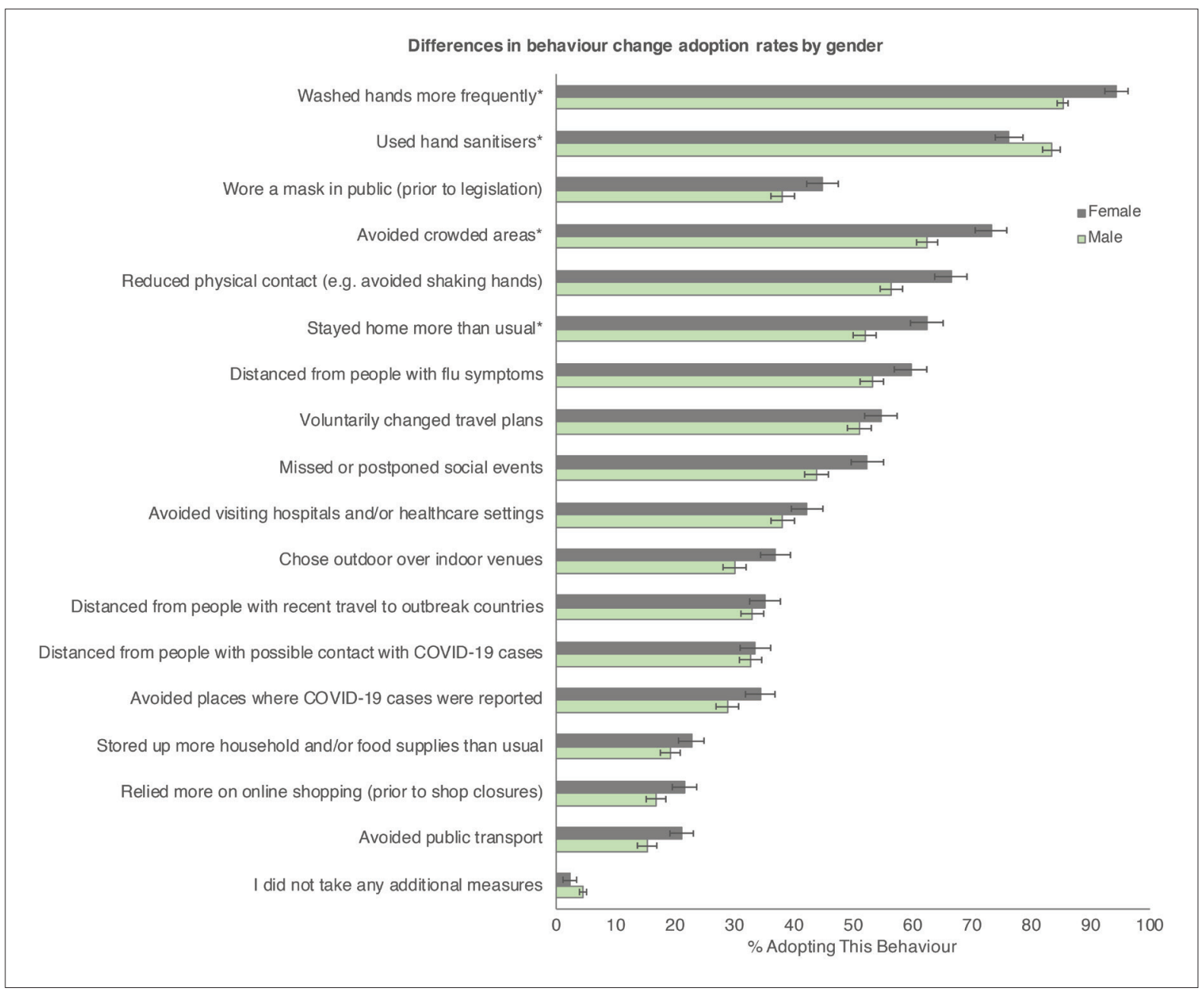

Fig. 1. Uptake of COVID-19 infection control measures as a function of gender. Asterisks indicate significance at $P<0.002$ (following Bonferroni corrections), and horizontal lines represent the $95 \%$ confidence intervals. 


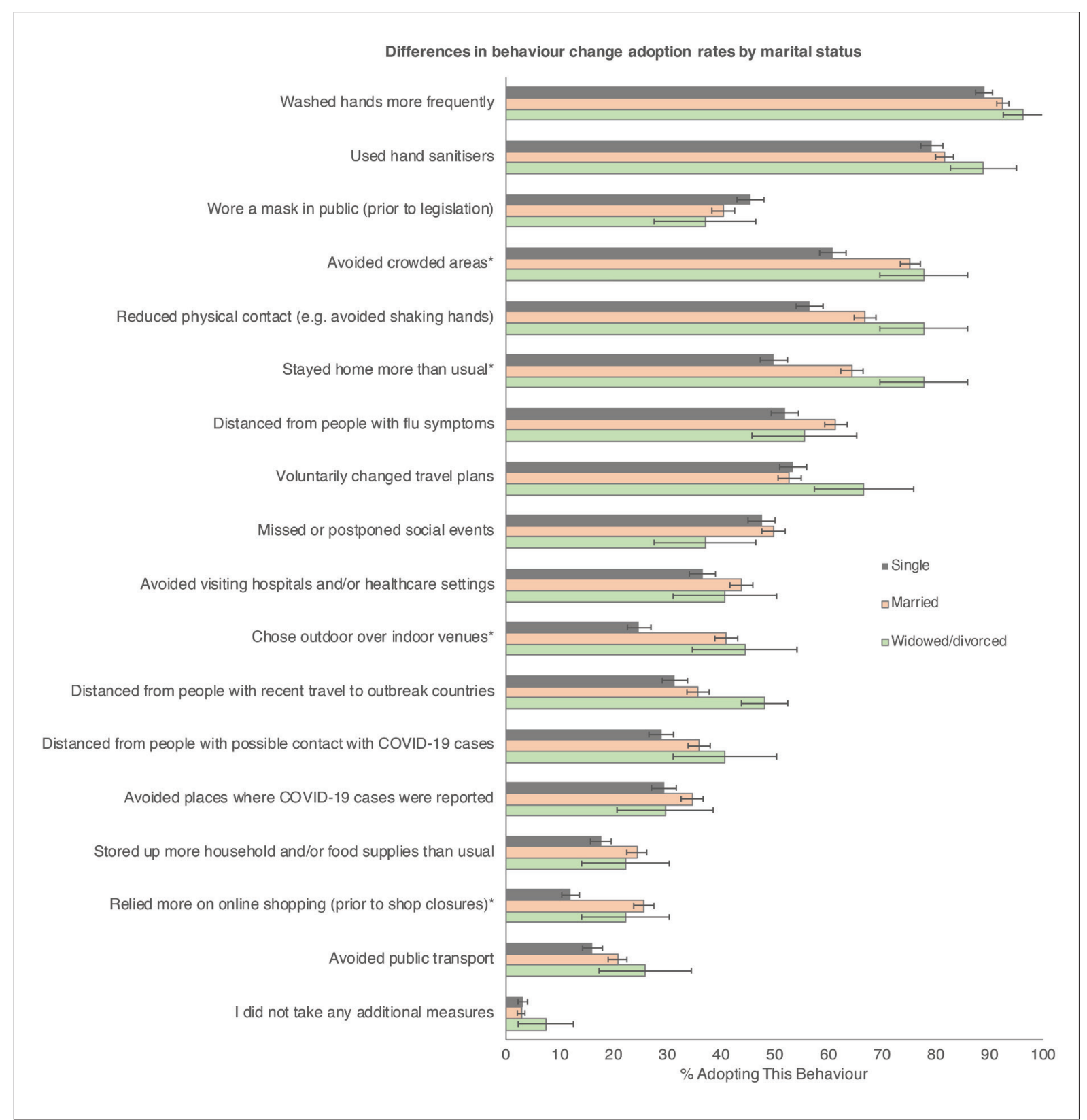

Fig. 2. Uptake of COVID-19 infection control measures as a function of marital status. Asterisks indicate significance at $P<0.002$ (following Bonferroni corrections), and horizontal lines represent the $95 \%$ confidence intervals.

and those who were married. When we distinguished between preventive (e.g. hand washing) and avoidant (e.g. avoiding crowded areas) behaviours, age emerged as an additional predictor for avoidant behaviours, with youths most likely to adopt mask-wearing.

Collectively, our results on gender and marital status replicate findings from previous infectious disease outbreaks ${ }^{3,13}$ and the current COVID-19 pandemic (based on both an international and a South Korean sample $\left.{ }^{7,9}\right)$. These findings echo a broader pattern of risk that has emerged in epidemiological research, whereby being women and being married has been linked to the reduced risk of disease and of all-cause mortality. ${ }^{14}$ Adding to this body of research, our findings highlight how being willing to adopt health-promoting behaviours during a pandemic may contribute to the resilience of these demographic groups.

Departing from prior research and popular belief, however, we found that age was inversely related to the take-up of preventive behaviours. In particular, 


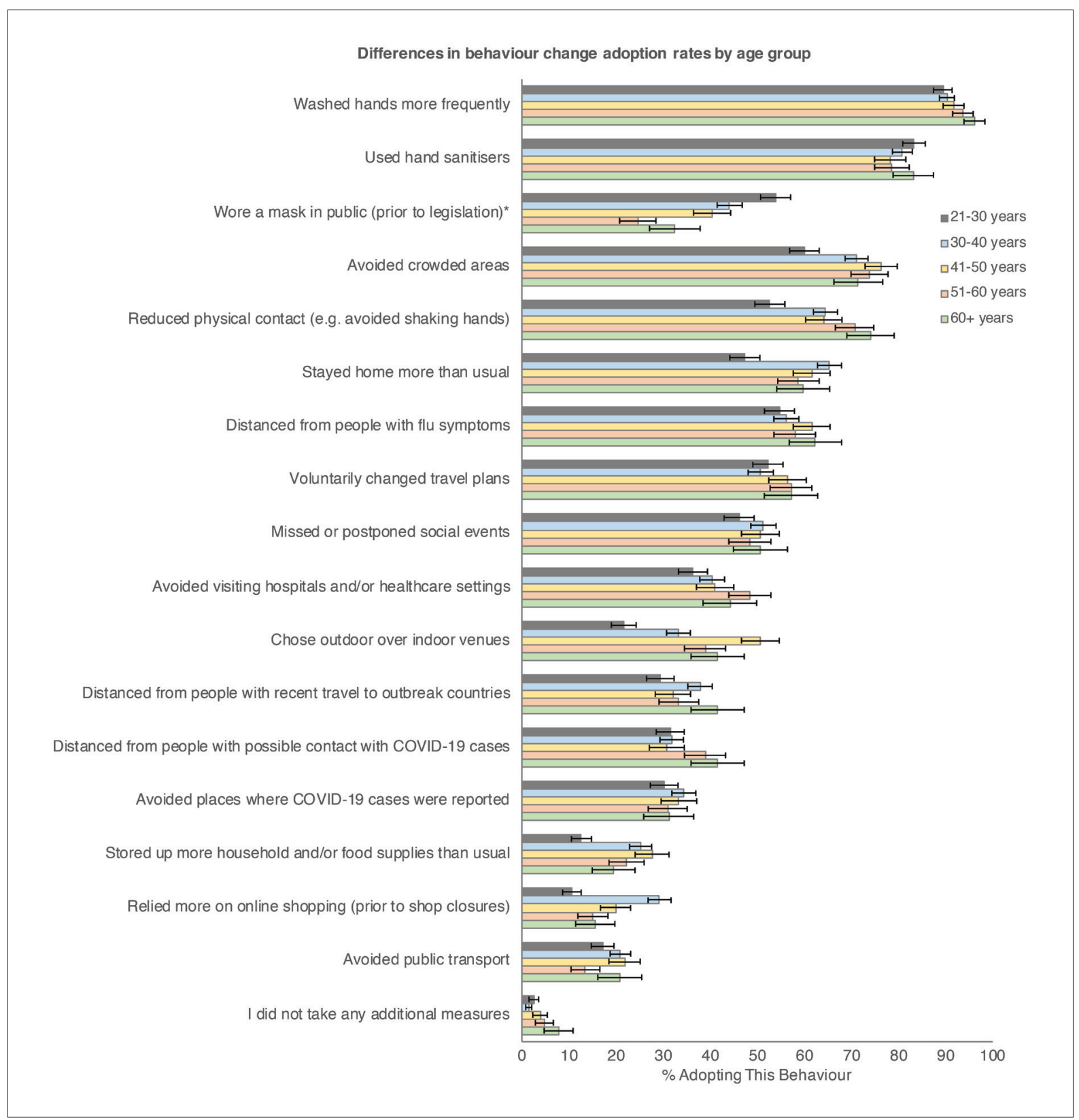

Fig. 3. Uptake of COVID-19 infection control measures as a function of age group. Asterisks indicate significance at $P<0.002$ (following Bonferroni corrections), and horizontal lines represent the $95 \%$ confidence intervals.

younger adults in our survey were more likely to wear masks than older adults, even before legislation stipulating that masks had to be worn in public. This finding is remarkable for several reasons. First, during SARS, older adults had been more likely to perform a range of preventive behaviours including maskwearing, handwashing, respiratory hygiene, the using of utensils, and washing after touching contaminated surfaces. ${ }^{3}$ Second, during the current outbreak, several high-profile events (e.g. coronavirus parties hosted by students) have resulted in the belief that youths are least likely to care about the outbreak, and thereby most likely to ignore infection control measures. Indeed, the Director-General of the World Health Organization released a statement telling youths that they were "not invincible", that "the virus could put (them) in hospital for weeks, or even kill (them)". ${ }^{15-17}$.

Rather than finding that young persons take on risky behaviours, however, we observed that this demographic group was most associated with mask- 
wearing. While this finding is counter-intuitive, it is in line with recent Hong Kong research whereby elderly participants - rather than the young - were least likely to worry about getting infected, and thus least likely to adopt protective behaviours. ${ }^{18}$ Additionally, young persons' ready adoption of mask-wearing may reflect a general willingness to embrace change and innovation, since mask-wearing had not previously been a norm in Singapore (as it had in countries like Japan ${ }^{19}$ ).

Beyond demographic predictors, we found that behavioural changes tracked the severity of the COVID-19 situation. Namely, participants adapted more aspects of their daily lives when the number of local COVID-19 cases increased, or as they grew more fearful of the situation. Correspondingly, healthy levels of COVID-19 fears may be necessary to support public health efforts. At the same time, other studies have linked the uptake of health-protective behaviours (e.g. hand-washing) to better mental health during the pandemic. ${ }^{20}$ In other words, it appears that while some fear is needed to encourage lifestyle modifications, the individual who proactively makes these modifications is more resilient to depression, anxiety and stress. ${ }^{20}$

\section{Policy implications}

Moving forward, our findings may contribute to the public health strategy in several ways. First, throughout the pandemic, government agencies have repeatedly noted how individuals have ignored official advisories. This phenomenon has been so widespread that the individuals have been nicknamed "covidiots" in the popular press - a portmanteau of coronavirus and idiot. Beyond naming and shaming, however, our research highlights characteristics that may predict noncompliance. This, in turn, will allow risk communication to be targeted: both in the current pandemic (for voluntary behavioural changes), and in the early phases of future pandemics (before measures such as maskwearing are made mandatory).

On the other hand, our findings also highlight which demographic groups may be most likely to respond when governments launch new infection control measures (for example, SafeEntry or the TraceTogether application for contact tracing). Extrapolating from our research, these initiatives - if perceived to be healthprotective - may be adopted first by women and those who are married. Correspondingly, the two demographic groups may be ideal for pilot trials or as advocates for the behaviours.

Finally, it is notable that across demographic groups, voluntary avoidant behaviours were less likely to be adopted than voluntary preventive behaviours. This could suggest the need for stronger measures (e.g. legislation) when public health agencies seek to increase avoidant behaviours to minimise activities deemed high-risk for COVID-19 transmission.

\section{Limitations}

In making these recommendations, we note that our study has several limitations. First, we relied on participants' self-reports, which may be vulnerable to memory or social desirability biases. Future research will thus need to explore whether our findings translate to actual behavioural changes during the pandemic, and to examine whether the frequency of behaviours (and not merely the uptake of behaviours) differs across demographic groups. Second, although our survey methodology captured behavioural changes at one particular time-point during the early phase of the pandemic, the recommendation of infection control measures is a moving target. In the case of maskwearing, for example, official advisories changed from masks not being needed, to being encouraged, to finally being mandated (as of 14 April 2020). Correspondingly, further research is needed to examine whether our findings continue to hold even as official advisories change and more measures (e.g. safe distancing, contact tracing applications) are implemented..$^{22}$ Third, we note that despite having a large sample size and a wide range of participant backgrounds, our final study sample was not representative of the national population. This may limit the generalisability of our results, and future research will need to examine whether our conclusions apply to under-sampled groups (e.g. those living in 1-3 room HDB flats).

\section{CONCLUSION}

We conducted the first Singapore-based study of behavioural changes during the COVID-19 pandemic. Although the scale of this crisis has been unprecedented and many uncertainties remain, many of our findings reinforce long-standing patterns of how demographic characteristics can predispose an individual to disease. In this case, the uptake of various preventive and avoidance measures can minimise COVID-19 infection. Moving forward, our findings provide a template by which official messaging can be tailored for health promotion.

\section{Acknowledgements}

This research was funded by a grant awarded from the JY Pillay Global Asia Programme (grant number: IG20-SG002). The authors would like to acknowledge Saw Young Ern and Edina Tan for assisting with this manuscript's preparation. 


\section{REFERENCES}

1. Tan THY, Toh MPHS, Vasoo S, et al. Coronavirus Disease 2019 (COVID-19): The Singapore Experience. A Review of the First Eight Months. Ann Acad Med Singap 2020;49:764-78.

2. Chen JI-P, Yap JC-H, Hsu LY, et al. COVID-19 and Singapore: From Early Response to Circuit Breaker. Ann Acad Med Singap 2020;49:561-72

3. Bish A, Michie S. Demographic and attitudinal determinants of protective behaviours during a pandemic: A review. Br J Health Psychol 2010;15:797-824.

4. Ferguson N, Laydon D, Nedjati Gilani G, et al. Report 9: Impact of Non-Pharmaceutical Interventions (NPIs) to Reduce COVID19 Mortality and Healthcare Demand. Imperial College London 2020. doi:10.25561/77482

5. Aledort JE, Lurie N, Wasserman J, et al. Non-pharmaceutical public health interventions for pandemic influenza: an evaluation of the evidence base. BMC Public Health 2007;7:208.

6. Yıldırım M, Geçer E, Akgül Ö. The impacts of vulnerability, perceived risk, and fear on preventive behaviours against COVID-19. Psychol Health Med 2021;26:35-43.

7. Lee M, You M. Psychological and Behavioral Responses in South Korea During the Early Stages of Coronavirus Disease 2019 (COVID-19). Int J Environ Res Public Health 2020;17:2977.

8. Harper CA, Satchell LP, Fido D, et al. Functional Fear Predicts Public Health Compliance in the COVID-19 Pandemic. Int J Ment Health Addict 2020:1-14.

9. Clark C, Davila A, Regis M, et al. Predictors of COVID-19 voluntary compliance behaviors: An international investigation. Glob Transit 2020;2:76-82.

10. Bogg T, Milad E. Demographic, personality, and social cognition correlates of coronavirus guideline adherence in a U.S. sample. Health Psychol Off J Div Health Psychol Am Psychol Assoc 2020;39:1026-36.

11. Liu JCJ, Tong EMW. The Relation Between Official WhatsAppDistributed COVID-19 News Exposure and Psychological Symptoms: Cross-Sectional Survey Study. J Med Internet Res 2020;22:e22142.

12. Zickfeld J, Schubert TW, Herting AK, et al. Correlates of Health-Protective Behavior During the Initial Days of the COVID-19 Outbreak in Norway. Front Psychol 2020;11:564083.
13. Moran KR, Valle SYD. A Meta-Analysis of the Association between Gender and Protective Behaviors in Response to Respiratory Epidemics and Pandemics. PLOS ONE 2016;11:e164541.

14. Wang $Y$, Jiao $Y$, Nie J, et al. Sex differences in the association between marital status and the risk of cardiovascular, cancer, and all-cause mortality: a systematic review and meta-analysis of 7,881,040 individuals. Glob Health Res Policy 2020;5:4

15. World Health Organization. WHO Director-General's opening remarks at the media briefing on COVID-19, 20 March 2020. Available at: https://www.who.int/dg/speeches/detail/who-directorgeneral-s-opening-remarks-at-the-media-briefing-on-covid-19---20march-2020. Accessed on 25 July 2020.

16. Gharzai LA, Beeler WH, Jagsi R. Playing Into Stereotypes: Engaging Millennials and Generation Z in the COVID-19 Pandemic Response. Adv Radiat Oncol 2020;5:679-81.

17. Jørgensen FJ, Bor A, Petersen MB. Compliance Without Fear: Predictors of Protective Behavior During the First Wave of the COVID-19 Pandemic. PsyArXiv 2020. doi:10.31234/osf.io/uzwgf

18. Chan EYY, Huang Z, Lo ESK, et al. Sociodemographic Predictors of Health Risk Perception, Attitude and Behavior Practices Associated with Health-Emergency Disaster Risk Management for Biological Hazards: The Case of COVID-19 Pandemic in Hong Kong, SAR China. Int J Environ Res Public Health 2020;17:3869.

19. Wada K, Oka-Ezoe K, Smith DR. Wearing face masks in public during the influenza season may reflect other positive hygiene practices in Japan. BMC Public Health 2012;12:1065.

20. Wang C, Pan R, Wan X, et al. A longitudinal study on the mental health of general population during the COVID-19 epidemic in China. Brain Behav Immun 2020;87:40-8

21. Ministry of Health, Singapore. Continued Stringent Implementation \& Enforcement Of Circuit Breaker Measures, 14 April 2020. Available at: https:/www.moh.gov.sg/news-highlights/details/ continued-stringent-implementation-enforcement-of-circuit-breakermeasures. Accessed on 30 July 2020.

22. Saw YE, Tan EY-Q, Liu JS, et al. Predicting Public Uptake of Digital Contact Tracing During the COVID-19 Pandemic: Results From a Nationwide Survey in Singapore. J Med Internet Res 2021;23:e24730. 